\title{
Tomographic renal cortical scintigraphy: Correlation with intravenous urography, computed tomography, ultrasonography, angiography, and nuclear magnetic resonance imaging
}

\author{
David A. Schultz ${ }^{1}$, Brahm Shapiro ${ }^{2}$, Marco Amendola ${ }^{2}$, Craig Sherman ${ }^{1}$, and Richard L. Wahl ${ }^{2}$ \\ ${ }^{1}$ New York Hospital-Cornell Medical Center, New York, New York, USA \\ ${ }^{2}$ The University of Michigan Medical Center, Ann Arbor, Michigan, USA
}

\begin{abstract}
This study evaluates single-photon renal tomoscintigraphy (SPECT) in the evaluation of renal masses and correlates this modality, where indicated, with computed tomography (CT), ultrasonography (US), angiography (ANGIO) and nuclear magnetic resonance imaging (NMR). Eight patients with renal cortical lesions detected on intravenous urography (IVP) were evaluated by SPECT and planar nuclear imaging using Tc-99m glucoheptonate (GH). Three of these patients were felt particularly likely to have renal tumors and were additionally evaluated with US, CT, ANGIO and NMR. The five patients with nodules on IVP that were not particularly suggestive of malignancy had functioning, benign, renal tissue accounting for their IVP lesions. Four of five were found by planar-GH nuclear imaging, five/five by SPECT-GH. In addition, SPECT-GH allowed better "confidence" in the normal renal tissue diagnosis in three/five cases. Of the three renal lesions that were highly suggestive of malignancy, two were hypernephromas and one was hypertrophied functioning cortical tissue. All three were correctly identified prospectively on SPECT$\mathrm{GH}$; however, one hypernephroma was missed on planarGH. NMR, CT, and ANGIO detected only one of two hypernephromas prospectively (US detected both); all four modalities incorrectly diagnosed the hypertrophied tissue suggestive of malignancy.
\end{abstract}

Key words: Hypernephromas - Renal cortex imaging - Columns of Bertin - Renal tumors

\section{Introduction}

The scintigraphic evaluation of suspected benign and malignant parenchymal lesions has been studied for many years. Until the early 1970 s, mercurial-labelled chlormerodrin was used extensively for anatomical and functional data (Pollack et al. 1974). However, the radiomercury labels had limitations: ${ }^{203} \mathrm{Hg}$ had unfavorable dosimetry; ${ }^{197} \mathrm{Hg}$ had a suboptimal energy (Daly et al. 1978). Subsequently, ${ }^{99 \mathrm{~m}} \mathrm{Tc}-$ labelled dimercaptosuccinic acid (DMSA) has been utilized (MacDonald et al. 1977; Daly et al). More recently, renal

Offprint requests to: David A. Schultz, M.D., Department of Radiology, St. Francis Hospital, 1018 South 13th Street, Escanaba, MI 49829, USA parenchymal imaging studies have generally utillzed ${ }^{99 \mathrm{~m}} \mathrm{Tc}-$ labelled glucoheptonate $(\mathrm{GH})$ in the evaluation of renal lesions (Leonard et al. 1979: O'Reilly et al. 1981; Teates et al. 1983). The latest advances in renal cortical imaging involve the use of single photon-emission computed tomography (SPECT). Williams and Parker (1982) used ${ }^{99 m}$ TcDMSA SPECT in a patient to evaluate a column of Bertin. Teates et al. (1983) utilized emission tomography with ${ }^{99 \mathrm{~m}} \mathrm{Tc}-\mathrm{GH}$ and showed superior diagnostic capabilities compared to standard planar renal parenchymal scintigraphy in two patients. Besides simple cysts and renal neoplasms, renal anatomical variations often necessitating further diagnostic evaluation after discovery on intravenous urography (IVP) include: dromedary hump, fetal lobulation, lobular compensatory hypertrophy, columns of Bertin, supra- and infrahilar cortical lips, and cortical nodules.

\section{Materials and methods}

Eight recent patients whose urograms were equivocal or suggestive of the presence of a renal mass were selected for this multimodality study. A suspicious lesion was determined by patients undergoing IVP [100 cc rapid bolus of meglumine diatrizoate (Hypaque - 50\%), with tomograms], and the lesions on the urogram were most likely due to neoplasm or other parenchymal process, i.e., not having the classic uroradiologic features of a benign cyst. All eight patients underwent planar and tomographic GH scintigraphy. Scintigrams were performed after a $10 \mathrm{mCi}$ intravenous bolus of Technetium 99m GH. For planar imaging, gamma camera was used with a wide viewfield and a lowenergy, all-purpose, parallel-hole collimator interfaced to a computer. A flow study was obtained at 2 -s intervals for $60 \mathrm{~s}$. Parallel-hole collimator posterior and posterior oblique views were obtained at $20 \mathrm{~min}$. At $2-3 \mathrm{~h}$, pinhole views were obtained in appropriate projections. Tomographic renal cortical imaging was performed at the same time as the early and delayed views without additional injection of radionuclide. Tomographic imaging was performed on a General Electric 400AT Anger Camera interfaced to the General Electric Star Computer System. For tomographic display, acquisitions were obtained in a $64 \times 64$ matrix (64 planar projections approximately every $6^{\circ}$, approximately 100,000 counts per $6 \mathrm{~mm}$ slice, imaging time of 

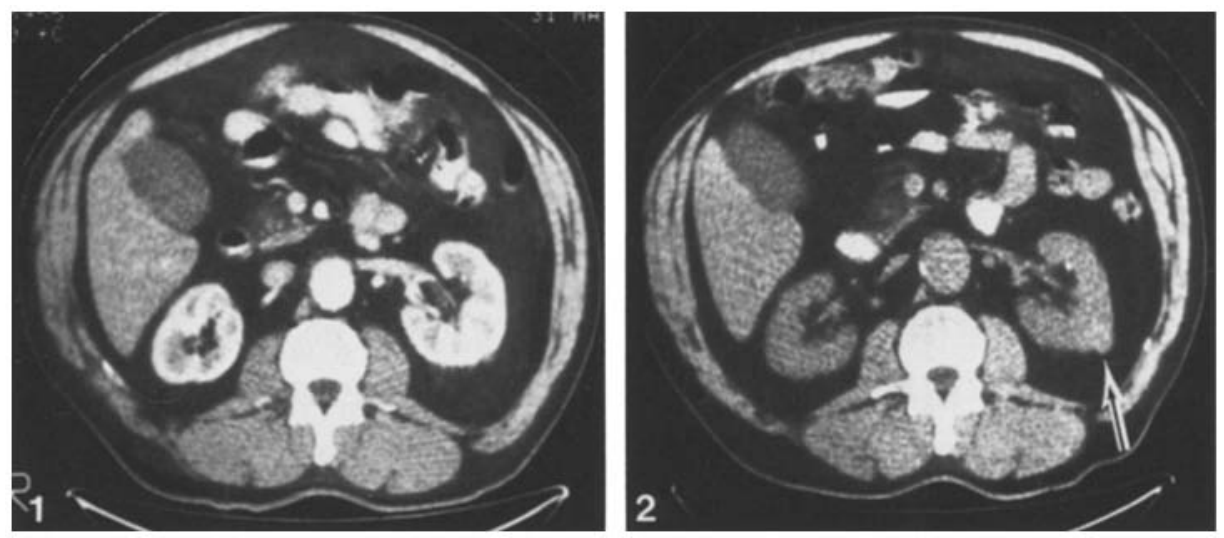

Fig. 1. Contrast-enhanced CT; no renal masses detected

Fig. 2. Non-contrast-enhanced CT at same renal level 6 months later; iso-intense cortical mass showing projection beyond cortex (arrow)

16 min). Back-projection and three-dimensional filtering yielded data satisfactory for sagittal, coronal, and transaxial formatting. In three of the eight patients, the intravenous urogram was more highly suggestive of renal neoplasm and in these three patients, ultrasound (US), computed tomography (CT), and angiography (ANGIO) were performed. The two patients with hypernephromas were also imaged using nuclear magnetic resonance (NMR). Ultrasound examination was performed using state-of-the-art real time (ATL) and gray-scale static B-mode scanning. CT was performed using a General Electric 8800 high-resolution tomographic scanner with intravenous contrast enhancement. ANGIO was performed in sterile fashion, using the Seldinger technique with selected renal arterial injections. NMR imaging was performed on a Diasonics unit operating at 0.35 TESLA $(\mathrm{TR}=$ repetition time - seconds, $\mathrm{TE}=$ time to echo - milliseconds; pulse sequences obtained: TR 0.5, TE 28 and 56; TR 1.0, TE 28 and 56; TR 1.5, TE 28 and 56 ; TR 2.0, TE 28 and 56).

\section{Results}

Five of the eight patients undergoing IVP had either cortical "bumps" or suspected cortical nodules or columns of Bertin. Of the five patients with cortical bumps or suspected columns of Bertin, four of five $(80 \%)$ showed "normal cortical tissue" on planar GH images. All five of five patients $(100 \%)$ showed normal cortical tissue on tomographic $\mathrm{GH}$ images. In addition, the tomographic GH images showed the functioning "bumps" better and with a higher degree of certainty and resolution (three out of five patients) than planar GH images. Tomographic GH scanning also resolved cortical scars (from chronic pyelonephritis on the IVP) and bowel activity (from biliary secretion in one patient) missed on planar GH imaging. Higher imaging modalities were not performed on these patients. These patients have done well in follow-up for 12-18 months without change on their IVPs. Of the three remaining patients suspected of having renal tumors, two hypernephromas were discovered. These patients had higher imaging modalities performed. In one patient, IVP showed a large, soft tissue mass arising from the lateral portion of the left kidney. Ultrasonography (US) revealed a solid renal mass, hypernephroma to be ruled out. CT revealed a large mass arising from the left kidney, which showed identical enhancement to the rest of the renal cortex; however, a hyper-

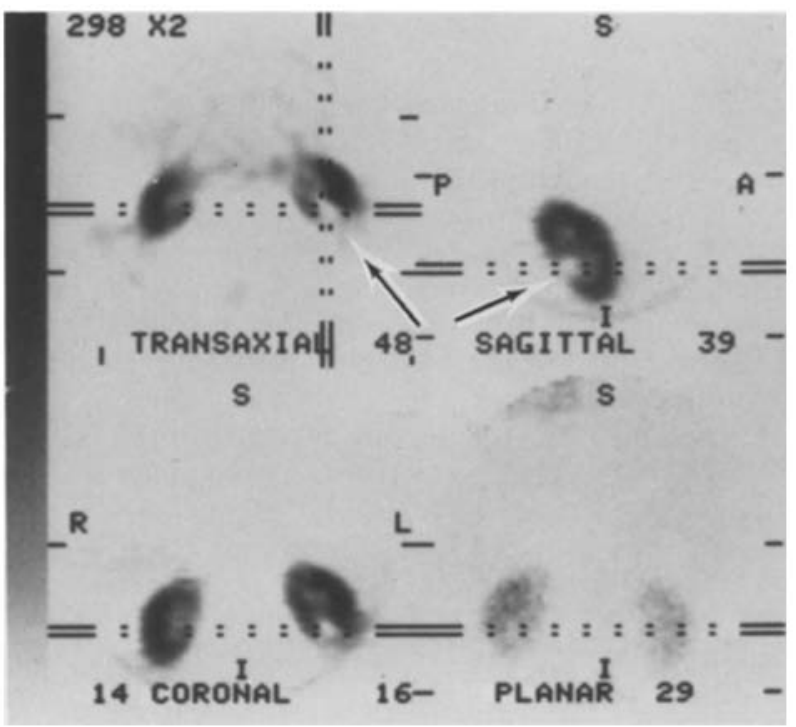

Fig. 3. Tomographic (SPECT) glucoheptonate $(\mathrm{GH})$ renal scan. Although the planar GH images were normal, SPECT GH showed a cortical defect corresponding to the renal mass on CT, seen on the transaxial and sagittal images (arrows)

hephroma could not be ruled out. ANGIO was performed and was interpreted as tortuosity and irregularity to the arteries feeding the soft tissue mass. The angiographic diagnosis was: postinflammatory changes versus hypernephroma. Both planar and tomographic GH scanning revealed that the large soft tissue mass arising from the left kidney took up the radiotracer and was diagnostic of benign cortical tissue, greatly hypertrophied since the right kidney and residual portions of the left kidney were shrunken from chronic pyelonephritis. This is the only patient with a highly suspicuous lesion on IVP that did not have NMR performed.

One patient with a large mass involving the lower portion of the right kidney discovered on IVP showed the classic uroradiographic appearance for a hypernephroma. US evaluation revealed a solid mass. The CT diagnosis was hypernephroma, as was the ANGIO diagnosis. On NMR, the infiltrated right lower pole neoplasm seemed to involve the renal hilar vein and inferior vena cava. Both planar and tomographic GH imaging showed lack of up- 
take in the lower portion of the right kidney, consistent with a mass.

In the last patient, the IVP, US and CT failed to detect a solid renal tumor (Fig. 1). Five months later, due to flank pain, the patient was reimaged with ultrasound. At this time, there was a new cortical bump. A CT scan was recommended which revealed a $1.1 \mathrm{~cm}$ cortical mass (Fig. 2). NMR failed to detect the lesion. At ANGIO, the suspected lesion was avascular and hypernephroma was not considered likely. This lesion seen on CT could not be identified on planar $\mathrm{GH}$ imaging; however, it was readily identifiable on tomographic GH (Fig. 3) scanning in both sagittal and transaxial projections. Surgery revealed a hypernephroma.

\section{Discussion}

In 43 patients with suggested masses identified on urograms (Pollack 1974), ${ }^{197} \mathrm{Hg}$-chlormerodrin was compared to arteriography. The renal cortical imaging correctly separated the pseudotumors from true tumors in all cases, and it was concluded that arteriography would only rarely be necessary to settle the question of true versus pseudomasses. This was especially true in patients with normal radionuclide studies. In a study (Leonard et al. 1979) comparing 41 patients undergoing IVP and GH scanning, the sensitivity for detecting renal mass lesions was significantly better with GH. They suggested GH scanning in the early evaluation of suspected renal mass lesions. O'Reilly et al. (1981), in utilizing $\mathrm{GH}$ arterial flow studies, detected no arterial flow in any of 14 patients with cysts and tumor circulation in 9 of 10 patients with hypernephromas. In 2 patients with necrotic masses, the nuclear arteriograms were avascular. Williams and Parker (1982) described in their case report how US failed to evaluate a hypertrophied column of Bertin, which was well seen on SPECT-GH.

Kass et al. (1983) described examples of renal malignancies diagnosed in patients with normal excretory urograms including tomography. They emphasized using another modality such as CT or US in patients highly suspected of having a renal mass. Charboneau et al. (1983) described the high incidence of renal malignancy in patients whose renal masses do not meet the sonographic criteria for benign simple cysts. Amis and Hartman (1984) list multiple diagnoses, such as lymphoma and abscess, which can be confused with simple renal cysts on US. The value of CT in diagnosis of renal lesions is well established (Magilner and Ostrum 1978), but there are indeterminate cases.

ANGIO was the classic approach to the diagnosis of hypernephroma; however, there is associated morbidity and mortality. High-resolution CT in many cases is superior to ANGIO in preoperative diagnosis and in staging of renal malignancy (Kam et al. 1981; Pillari et al. 1981; Weyman et al. 1980). Although most hypernephromas are hypervascular, a small percentage of hypernephromas are normovascular or hypovascular relative to the surrounding normal renal parenchyma. In comparing US to ANGIO, the echogeneity was found to correlate very well with the degree of neovascularity (Ladwig et al. 1981; Maklad et al. 1977). $\mathrm{CT}$ is approximately equally as accurate as ANGIO in detecting main renal vein and vena caval involvement (Weyman et al. 1980).

NMR has been shown to be of value not only in the evaluation of renal cysts and masses, but in patients with other types of renal pathology. NMR has been shown to differentiate simple renal cysts from other renal masses, although there is overlap between solid renal masses and hemorrhagic cysts. In addition, extension of tumor thrombus into the inferior vena cava can be demonstrated on NMR (Hricak et al. 1983a, b).

In conclusion, tomographic GH scanning has a higher sensitivity, resolution and certainty than planar GH scanning for detecting small renal tumors, scars, and cysts. Of course, a cold defect on a GH scan must be correlated with US to rule out a benign cystic lesion. Lesions on GH scanning that show uptake of the radiotracer can be diagnosed as normal functioning cortical tissue. This is best seen in the patient where the IVP was suggestive of a tumor and the US, CT, and ANGIO were interpreted as "cannot rule out hypernephroma", but GH scanning revealed functioning cortical tissue (the patient's scheduled nephrectomy was cancelled). In one patient with a hypernephroma, US and CT originally missed the tumor. Even after discovery on later US and CT, the lesion could not be identified on ANGIO, NMR, or planar GH scanning. Tomographic GH scanning did identify this $1.1 \mathrm{~cm}$ tumor.

The future role of NMR imaging is yet to be determined and should improve as better pulse sequences are discovered. Tomographic GH can significantly improve the diagnostic evaluation of renal masses and has the potential to obviate unnecessary invasive procedures. In this small series of eight patients, it was the only modality without false-positives or false-negatives for a renal cell carcinoma. In particular, it is ideally able to evaluate cortical bumps, nodules, and columns of Bertin.

\section{References}

Amis ES Jr, Hartman DS (1984) Renal ultrasonography 1984: a practical overview. Radiol Clin North Am 22:315-329

Charboneau JW, Hattery RR, Ernst EC III, James EM, Williamson B Jr, Hartman GW (1983) Spectrum of sonographic findings in 125 renal masses other than benign simple cyst. AJR 140:87-94

Daly MJ, Milutinovic J, Rudd TG, Phillips LA, Fialkow PJ (1978) The normal $99 \mathrm{mTc}-\mathrm{DMSA}$ renal image. Radiology 128:701-704

Hricak H, Crooks L, Sheldon P, Kaufman L (1983a) Nuclear magnetic resonance imaging of the kidney. Radiology 146:425-532

Hricak H, Williams RD, Moon KL Jr, Moss AA, Alpers C, Crooks LE, Kaufman L (1983b) Nuclear magnetic resonance imaging of the kidney: renal masses. Radiology 147:765-772

Kam J, Sandler CM, Benson GS (1981) Angiography in diagnosis of renal tumors - current concepts. Urology 19:100-106

Kass DA, Hricak H, Davidson AJ (1983) Renal malignancies with normal excretory urograms. AJR $141: 731-734$

Ladwig SH, Jackson D, Older RA, Morgan CL (1981) Ultrasonıc, angiographic, and pathologic correlation of noncystic-appearing renal masses. Urology 17:204 209

Leonard JC, Allen EW, Goin J, Smith CW (1979) Renal cortical imaging and the detection of renal mass lesions. $\mathrm{J}$ Nucl Med $20: 1018-1022$

MacDonald AF, Keyes WI, Mallard JR, Steyn JH (1977) Diagnostic value of computerised isotopic section renal scanning. Eur Urol 3:289-291

Magilner AD, Ostrum BJ (1978) Computed tomography in the diagnosis of renal masses. Radiology 126:715-718

Maklad NF, Chuang VP, Doust BD, Cho KJ, Curran JE (1977) Ultrasonic characterization of solid renal lesions: echographic, 
angiographic and pathologic correlation. Radiology 123: 733-739

O'Reilly PH, Osborn DE, Testa HJ, Asbury DL, Best JJK, Barnard RJ (1981) Renal imaging: a comparison of radionuclide, ultrasound, and computed tomograhic scanning in investigation of renal space-occupying lesions. Br Med J 282:943-945

Pillari G, Lee WJ, Kumari S, Chen M, Abrams HJ, Buchbinder $M$, Sutton AP (1981) CT and angiographic correlates: surgical image of renal mass lesions. Urology 17:296-299

Pollack HM, Edell S, Morales JO (1974) Radionuclide imaging in renal pseudotumors. Radiology 111:639-644

Teates CD, Croft BY, Brenbridge AG, Bray ST, Williamson RJ
(1983) Emission tomography of the kidney. South Med J $76: 1499-1502$

Weyman PJ, McClennan BL, Stanley RJ, Levitt RG, Sagel SS (1980) Comparison of computed tomography and angiography in the evaluation of renal cell carcinoma. Radiology $137: 417-424$

Williams ED, Parker C (1982) Kidney pseudotumour diagnosed by emission computed tomography. Br Med J 285:1379-1380

Received February 9, 1985 / July 6, 1985 